\title{
Phase Transition Results for Three Ramsey-Like Theorems
}

\author{
Florian Pelupessy
}

\begin{abstract}
We classify a sharp phase transition threshold for Friedman's finite adjacent Ramsey theorem. We extend the method for showing this result to two previous classifications involving Ramsey theorem variants: the ParisHarrington theorem and the Kanamori-McAloon theorem. We also provide tools to remove ad hoc arguments from the proofs of phase transition results as much as currently possible.
\end{abstract}

Phase transitions in logic are a recent development in unprovability. The general program, started by Andreas Weiermann, is to classify parameter functions $f: \mathbb{N} \rightarrow \mathbb{N}$ according to the provability of a parameterized theorem $\varphi_{f}$ in a theory $T$. We study these transitions with the goal of gaining a better understanding of unprovability. More details on this program, with an overview of related publications, can be found at Weiermann [16].

In this paper we examine the transition results for three Ramsey theorem variants: Friedman's finite adjacent Ramsey theorem, the Paris-Harrington theorem, and the Kanamori-McAloon theorem. The latter two of these have been studied previously in Weiermann [15] and Carlucci, Lee, and Weiermann [4], but the methods used in the present paper are a natural continuation of the method for the adjacent Ramsey theorem. The emphasis of this method is on connecting the variants $\varphi_{k}$ of the theorem for constant functions $k$ with the classification $f$ according to the provability of $\varphi_{f}$. Furthermore, this proof method does not depend on whether the original nonparameterized version of the theorem was shown using proof/recursion theory or model-theoretic constructions.

Received March 20, 2013; accepted October 29, 2013

First published online January 6, 2016

2010 Mathematics Subject Classification: Primary 03F30; Secondary 03D20, 03H15

Keywords: finite adjacent Ramsey, Paris-Harrington, Kanamori-McAloon, Ramsey theory, unprovability, independence, phase transitions, Peano arithmetic

(C) 2016 by University of Notre Dame 10.1215/00294527-3452807 


\title{
Phase Transition Results for Three Ramsey-Like Theorems
}

\author{
Florian Pelupessy
}

\begin{abstract}
We classify a sharp phase transition threshold for Friedman's finite adjacent Ramsey theorem. We extend the method for showing this result to two previous classifications involving Ramsey theorem variants: the ParisHarrington theorem and the Kanamori-McAloon theorem. We also provide tools to remove ad hoc arguments from the proofs of phase transition results as much as currently possible.
\end{abstract}

Phase transitions in logic are a recent development in unprovability. The general program, started by Andreas Weiermann, is to classify parameter functions $f: \mathbb{N} \rightarrow \mathbb{N}$ according to the provability of a parameterized theorem $\varphi_{f}$ in a theory $T$. We study these transitions with the goal of gaining a better understanding of unprovability. More details on this program, with an overview of related publications, can be found at Weiermann [16].

In this paper we examine the transition results for three Ramsey theorem variants: Friedman's finite adjacent Ramsey theorem, the Paris-Harrington theorem, and the Kanamori-McAloon theorem. The latter two of these have been studied previously in Weiermann [15] and Carlucci, Lee, and Weiermann [4], but the methods used in the present paper are a natural continuation of the method for the adjacent Ramsey theorem. The emphasis of this method is on connecting the variants $\varphi_{k}$ of the theorem for constant functions $k$ with the classification $f$ according to the provability of $\varphi_{f}$. Furthermore, this proof method does not depend on whether the original nonparameterized version of the theorem was shown using proof/recursion theory or model-theoretic constructions.

Received March 20, 2013; accepted October 29, 2013

First published online January 6, 2016

2010 Mathematics Subject Classification: Primary 03F30; Secondary 03D20, 03H15

Keywords: finite adjacent Ramsey, Paris-Harrington, Kanamori-McAloon, Ramsey theory, unprovability, independence, phase transitions, Peano arithmetic

(C) 2016 by University of Notre Dame 10.1215/00294527-3452807 


\title{
Phase Transition Results for Three Ramsey-Like Theorems
}

\author{
Florian Pelupessy
}

\begin{abstract}
We classify a sharp phase transition threshold for Friedman's finite adjacent Ramsey theorem. We extend the method for showing this result to two previous classifications involving Ramsey theorem variants: the ParisHarrington theorem and the Kanamori-McAloon theorem. We also provide tools to remove ad hoc arguments from the proofs of phase transition results as much as currently possible.
\end{abstract}

Phase transitions in logic are a recent development in unprovability. The general program, started by Andreas Weiermann, is to classify parameter functions $f: \mathbb{N} \rightarrow \mathbb{N}$ according to the provability of a parameterized theorem $\varphi_{f}$ in a theory $T$. We study these transitions with the goal of gaining a better understanding of unprovability. More details on this program, with an overview of related publications, can be found at Weiermann [16].

In this paper we examine the transition results for three Ramsey theorem variants: Friedman's finite adjacent Ramsey theorem, the Paris-Harrington theorem, and the Kanamori-McAloon theorem. The latter two of these have been studied previously in Weiermann [15] and Carlucci, Lee, and Weiermann [4], but the methods used in the present paper are a natural continuation of the method for the adjacent Ramsey theorem. The emphasis of this method is on connecting the variants $\varphi_{k}$ of the theorem for constant functions $k$ with the classification $f$ according to the provability of $\varphi_{f}$. Furthermore, this proof method does not depend on whether the original nonparameterized version of the theorem was shown using proof/recursion theory or model-theoretic constructions.

Received March 20, 2013; accepted October 29, 2013

First published online January 6, 2016

2010 Mathematics Subject Classification: Primary 03F30; Secondary 03D20, 03H15

Keywords: finite adjacent Ramsey, Paris-Harrington, Kanamori-McAloon, Ramsey theory, unprovability, independence, phase transitions, Peano arithmetic

(C) 2016 by University of Notre Dame 10.1215/00294527-3452807 


\title{
Phase Transition Results for Three Ramsey-Like Theorems
}

\author{
Florian Pelupessy
}

\begin{abstract}
We classify a sharp phase transition threshold for Friedman's finite adjacent Ramsey theorem. We extend the method for showing this result to two previous classifications involving Ramsey theorem variants: the ParisHarrington theorem and the Kanamori-McAloon theorem. We also provide tools to remove ad hoc arguments from the proofs of phase transition results as much as currently possible.
\end{abstract}

Phase transitions in logic are a recent development in unprovability. The general program, started by Andreas Weiermann, is to classify parameter functions $f: \mathbb{N} \rightarrow \mathbb{N}$ according to the provability of a parameterized theorem $\varphi_{f}$ in a theory $T$. We study these transitions with the goal of gaining a better understanding of unprovability. More details on this program, with an overview of related publications, can be found at Weiermann [16].

In this paper we examine the transition results for three Ramsey theorem variants: Friedman's finite adjacent Ramsey theorem, the Paris-Harrington theorem, and the Kanamori-McAloon theorem. The latter two of these have been studied previously in Weiermann [15] and Carlucci, Lee, and Weiermann [4], but the methods used in the present paper are a natural continuation of the method for the adjacent Ramsey theorem. The emphasis of this method is on connecting the variants $\varphi_{k}$ of the theorem for constant functions $k$ with the classification $f$ according to the provability of $\varphi_{f}$. Furthermore, this proof method does not depend on whether the original nonparameterized version of the theorem was shown using proof/recursion theory or model-theoretic constructions.

Received March 20, 2013; accepted October 29, 2013

First published online January 6, 2016

2010 Mathematics Subject Classification: Primary 03F30; Secondary 03D20, 03H15

Keywords: finite adjacent Ramsey, Paris-Harrington, Kanamori-McAloon, Ramsey theory, unprovability, independence, phase transitions, Peano arithmetic

(C) 2016 by University of Notre Dame 10.1215/00294527-3452807 


\title{
Phase Transition Results for Three Ramsey-Like Theorems
}

\author{
Florian Pelupessy
}

\begin{abstract}
We classify a sharp phase transition threshold for Friedman's finite adjacent Ramsey theorem. We extend the method for showing this result to two previous classifications involving Ramsey theorem variants: the ParisHarrington theorem and the Kanamori-McAloon theorem. We also provide tools to remove ad hoc arguments from the proofs of phase transition results as much as currently possible.
\end{abstract}

Phase transitions in logic are a recent development in unprovability. The general program, started by Andreas Weiermann, is to classify parameter functions $f: \mathbb{N} \rightarrow \mathbb{N}$ according to the provability of a parameterized theorem $\varphi_{f}$ in a theory $T$. We study these transitions with the goal of gaining a better understanding of unprovability. More details on this program, with an overview of related publications, can be found at Weiermann [16].

In this paper we examine the transition results for three Ramsey theorem variants: Friedman's finite adjacent Ramsey theorem, the Paris-Harrington theorem, and the Kanamori-McAloon theorem. The latter two of these have been studied previously in Weiermann [15] and Carlucci, Lee, and Weiermann [4], but the methods used in the present paper are a natural continuation of the method for the adjacent Ramsey theorem. The emphasis of this method is on connecting the variants $\varphi_{k}$ of the theorem for constant functions $k$ with the classification $f$ according to the provability of $\varphi_{f}$. Furthermore, this proof method does not depend on whether the original nonparameterized version of the theorem was shown using proof/recursion theory or model-theoretic constructions.

Received March 20, 2013; accepted October 29, 2013

First published online January 6, 2016

2010 Mathematics Subject Classification: Primary 03F30; Secondary 03D20, 03H15

Keywords: finite adjacent Ramsey, Paris-Harrington, Kanamori-McAloon, Ramsey theory, unprovability, independence, phase transitions, Peano arithmetic

(C) 2016 by University of Notre Dame 10.1215/00294527-3452807 


\title{
Phase Transition Results for Three Ramsey-Like Theorems
}

\author{
Florian Pelupessy
}

\begin{abstract}
We classify a sharp phase transition threshold for Friedman's finite adjacent Ramsey theorem. We extend the method for showing this result to two previous classifications involving Ramsey theorem variants: the ParisHarrington theorem and the Kanamori-McAloon theorem. We also provide tools to remove ad hoc arguments from the proofs of phase transition results as much as currently possible.
\end{abstract}

Phase transitions in logic are a recent development in unprovability. The general program, started by Andreas Weiermann, is to classify parameter functions $f: \mathbb{N} \rightarrow \mathbb{N}$ according to the provability of a parameterized theorem $\varphi_{f}$ in a theory $T$. We study these transitions with the goal of gaining a better understanding of unprovability. More details on this program, with an overview of related publications, can be found at Weiermann [16].

In this paper we examine the transition results for three Ramsey theorem variants: Friedman's finite adjacent Ramsey theorem, the Paris-Harrington theorem, and the Kanamori-McAloon theorem. The latter two of these have been studied previously in Weiermann [15] and Carlucci, Lee, and Weiermann [4], but the methods used in the present paper are a natural continuation of the method for the adjacent Ramsey theorem. The emphasis of this method is on connecting the variants $\varphi_{k}$ of the theorem for constant functions $k$ with the classification $f$ according to the provability of $\varphi_{f}$. Furthermore, this proof method does not depend on whether the original nonparameterized version of the theorem was shown using proof/recursion theory or model-theoretic constructions.

Received March 20, 2013; accepted October 29, 2013

First published online January 6, 2016

2010 Mathematics Subject Classification: Primary 03F30; Secondary 03D20, 03H15

Keywords: finite adjacent Ramsey, Paris-Harrington, Kanamori-McAloon, Ramsey theory, unprovability, independence, phase transitions, Peano arithmetic

(C) 2016 by University of Notre Dame 10.1215/00294527-3452807 


\title{
Phase Transition Results for Three Ramsey-Like Theorems
}

\author{
Florian Pelupessy
}

\begin{abstract}
We classify a sharp phase transition threshold for Friedman's finite adjacent Ramsey theorem. We extend the method for showing this result to two previous classifications involving Ramsey theorem variants: the ParisHarrington theorem and the Kanamori-McAloon theorem. We also provide tools to remove ad hoc arguments from the proofs of phase transition results as much as currently possible.
\end{abstract}

Phase transitions in logic are a recent development in unprovability. The general program, started by Andreas Weiermann, is to classify parameter functions $f: \mathbb{N} \rightarrow \mathbb{N}$ according to the provability of a parameterized theorem $\varphi_{f}$ in a theory $T$. We study these transitions with the goal of gaining a better understanding of unprovability. More details on this program, with an overview of related publications, can be found at Weiermann [16].

In this paper we examine the transition results for three Ramsey theorem variants: Friedman's finite adjacent Ramsey theorem, the Paris-Harrington theorem, and the Kanamori-McAloon theorem. The latter two of these have been studied previously in Weiermann [15] and Carlucci, Lee, and Weiermann [4], but the methods used in the present paper are a natural continuation of the method for the adjacent Ramsey theorem. The emphasis of this method is on connecting the variants $\varphi_{k}$ of the theorem for constant functions $k$ with the classification $f$ according to the provability of $\varphi_{f}$. Furthermore, this proof method does not depend on whether the original nonparameterized version of the theorem was shown using proof/recursion theory or model-theoretic constructions.

Received March 20, 2013; accepted October 29, 2013

First published online January 6, 2016

2010 Mathematics Subject Classification: Primary 03F30; Secondary 03D20, 03H15

Keywords: finite adjacent Ramsey, Paris-Harrington, Kanamori-McAloon, Ramsey theory, unprovability, independence, phase transitions, Peano arithmetic

(C) 2016 by University of Notre Dame 10.1215/00294527-3452807 


\title{
Phase Transition Results for Three Ramsey-Like Theorems
}

\author{
Florian Pelupessy
}

\begin{abstract}
We classify a sharp phase transition threshold for Friedman's finite adjacent Ramsey theorem. We extend the method for showing this result to two previous classifications involving Ramsey theorem variants: the ParisHarrington theorem and the Kanamori-McAloon theorem. We also provide tools to remove ad hoc arguments from the proofs of phase transition results as much as currently possible.
\end{abstract}

Phase transitions in logic are a recent development in unprovability. The general program, started by Andreas Weiermann, is to classify parameter functions $f: \mathbb{N} \rightarrow \mathbb{N}$ according to the provability of a parameterized theorem $\varphi_{f}$ in a theory $T$. We study these transitions with the goal of gaining a better understanding of unprovability. More details on this program, with an overview of related publications, can be found at Weiermann [16].

In this paper we examine the transition results for three Ramsey theorem variants: Friedman's finite adjacent Ramsey theorem, the Paris-Harrington theorem, and the Kanamori-McAloon theorem. The latter two of these have been studied previously in Weiermann [15] and Carlucci, Lee, and Weiermann [4], but the methods used in the present paper are a natural continuation of the method for the adjacent Ramsey theorem. The emphasis of this method is on connecting the variants $\varphi_{k}$ of the theorem for constant functions $k$ with the classification $f$ according to the provability of $\varphi_{f}$. Furthermore, this proof method does not depend on whether the original nonparameterized version of the theorem was shown using proof/recursion theory or model-theoretic constructions.

Received March 20, 2013; accepted October 29, 2013

First published online January 6, 2016

2010 Mathematics Subject Classification: Primary 03F30; Secondary 03D20, 03H15

Keywords: finite adjacent Ramsey, Paris-Harrington, Kanamori-McAloon, Ramsey theory, unprovability, independence, phase transitions, Peano arithmetic

(C) 2016 by University of Notre Dame 10.1215/00294527-3452807 


\title{
Phase Transition Results for Three Ramsey-Like Theorems
}

\author{
Florian Pelupessy
}

\begin{abstract}
We classify a sharp phase transition threshold for Friedman's finite adjacent Ramsey theorem. We extend the method for showing this result to two previous classifications involving Ramsey theorem variants: the ParisHarrington theorem and the Kanamori-McAloon theorem. We also provide tools to remove ad hoc arguments from the proofs of phase transition results as much as currently possible.
\end{abstract}

Phase transitions in logic are a recent development in unprovability. The general program, started by Andreas Weiermann, is to classify parameter functions $f: \mathbb{N} \rightarrow \mathbb{N}$ according to the provability of a parameterized theorem $\varphi_{f}$ in a theory $T$. We study these transitions with the goal of gaining a better understanding of unprovability. More details on this program, with an overview of related publications, can be found at Weiermann [16].

In this paper we examine the transition results for three Ramsey theorem variants: Friedman's finite adjacent Ramsey theorem, the Paris-Harrington theorem, and the Kanamori-McAloon theorem. The latter two of these have been studied previously in Weiermann [15] and Carlucci, Lee, and Weiermann [4], but the methods used in the present paper are a natural continuation of the method for the adjacent Ramsey theorem. The emphasis of this method is on connecting the variants $\varphi_{k}$ of the theorem for constant functions $k$ with the classification $f$ according to the provability of $\varphi_{f}$. Furthermore, this proof method does not depend on whether the original nonparameterized version of the theorem was shown using proof/recursion theory or model-theoretic constructions.

Received March 20, 2013; accepted October 29, 2013

First published online January 6, 2016

2010 Mathematics Subject Classification: Primary 03F30; Secondary 03D20, 03H15

Keywords: finite adjacent Ramsey, Paris-Harrington, Kanamori-McAloon, Ramsey theory, unprovability, independence, phase transitions, Peano arithmetic

(C) 2016 by University of Notre Dame 10.1215/00294527-3452807 


\title{
Phase Transition Results for Three Ramsey-Like Theorems
}

\author{
Florian Pelupessy
}

\begin{abstract}
We classify a sharp phase transition threshold for Friedman's finite adjacent Ramsey theorem. We extend the method for showing this result to two previous classifications involving Ramsey theorem variants: the ParisHarrington theorem and the Kanamori-McAloon theorem. We also provide tools to remove ad hoc arguments from the proofs of phase transition results as much as currently possible.
\end{abstract}

Phase transitions in logic are a recent development in unprovability. The general program, started by Andreas Weiermann, is to classify parameter functions $f: \mathbb{N} \rightarrow \mathbb{N}$ according to the provability of a parameterized theorem $\varphi_{f}$ in a theory $T$. We study these transitions with the goal of gaining a better understanding of unprovability. More details on this program, with an overview of related publications, can be found at Weiermann [16].

In this paper we examine the transition results for three Ramsey theorem variants: Friedman's finite adjacent Ramsey theorem, the Paris-Harrington theorem, and the Kanamori-McAloon theorem. The latter two of these have been studied previously in Weiermann [15] and Carlucci, Lee, and Weiermann [4], but the methods used in the present paper are a natural continuation of the method for the adjacent Ramsey theorem. The emphasis of this method is on connecting the variants $\varphi_{k}$ of the theorem for constant functions $k$ with the classification $f$ according to the provability of $\varphi_{f}$. Furthermore, this proof method does not depend on whether the original nonparameterized version of the theorem was shown using proof/recursion theory or model-theoretic constructions.

Received March 20, 2013; accepted October 29, 2013

First published online January 6, 2016

2010 Mathematics Subject Classification: Primary 03F30; Secondary 03D20, 03H15

Keywords: finite adjacent Ramsey, Paris-Harrington, Kanamori-McAloon, Ramsey theory, unprovability, independence, phase transitions, Peano arithmetic

(C) 2016 by University of Notre Dame 10.1215/00294527-3452807 


\title{
Phase Transition Results for Three Ramsey-Like Theorems
}

\author{
Florian Pelupessy
}

\begin{abstract}
We classify a sharp phase transition threshold for Friedman's finite adjacent Ramsey theorem. We extend the method for showing this result to two previous classifications involving Ramsey theorem variants: the ParisHarrington theorem and the Kanamori-McAloon theorem. We also provide tools to remove ad hoc arguments from the proofs of phase transition results as much as currently possible.
\end{abstract}

Phase transitions in logic are a recent development in unprovability. The general program, started by Andreas Weiermann, is to classify parameter functions $f: \mathbb{N} \rightarrow \mathbb{N}$ according to the provability of a parameterized theorem $\varphi_{f}$ in a theory $T$. We study these transitions with the goal of gaining a better understanding of unprovability. More details on this program, with an overview of related publications, can be found at Weiermann [16].

In this paper we examine the transition results for three Ramsey theorem variants: Friedman's finite adjacent Ramsey theorem, the Paris-Harrington theorem, and the Kanamori-McAloon theorem. The latter two of these have been studied previously in Weiermann [15] and Carlucci, Lee, and Weiermann [4], but the methods used in the present paper are a natural continuation of the method for the adjacent Ramsey theorem. The emphasis of this method is on connecting the variants $\varphi_{k}$ of the theorem for constant functions $k$ with the classification $f$ according to the provability of $\varphi_{f}$. Furthermore, this proof method does not depend on whether the original nonparameterized version of the theorem was shown using proof/recursion theory or model-theoretic constructions.

Received March 20, 2013; accepted October 29, 2013

First published online January 6, 2016

2010 Mathematics Subject Classification: Primary 03F30; Secondary 03D20, 03H15

Keywords: finite adjacent Ramsey, Paris-Harrington, Kanamori-McAloon, Ramsey theory, unprovability, independence, phase transitions, Peano arithmetic

(C) 2016 by University of Notre Dame 10.1215/00294527-3452807 


\title{
Phase Transition Results for Three Ramsey-Like Theorems
}

\author{
Florian Pelupessy
}

\begin{abstract}
We classify a sharp phase transition threshold for Friedman's finite adjacent Ramsey theorem. We extend the method for showing this result to two previous classifications involving Ramsey theorem variants: the ParisHarrington theorem and the Kanamori-McAloon theorem. We also provide tools to remove ad hoc arguments from the proofs of phase transition results as much as currently possible.
\end{abstract}

Phase transitions in logic are a recent development in unprovability. The general program, started by Andreas Weiermann, is to classify parameter functions $f: \mathbb{N} \rightarrow \mathbb{N}$ according to the provability of a parameterized theorem $\varphi_{f}$ in a theory $T$. We study these transitions with the goal of gaining a better understanding of unprovability. More details on this program, with an overview of related publications, can be found at Weiermann [16].

In this paper we examine the transition results for three Ramsey theorem variants: Friedman's finite adjacent Ramsey theorem, the Paris-Harrington theorem, and the Kanamori-McAloon theorem. The latter two of these have been studied previously in Weiermann [15] and Carlucci, Lee, and Weiermann [4], but the methods used in the present paper are a natural continuation of the method for the adjacent Ramsey theorem. The emphasis of this method is on connecting the variants $\varphi_{k}$ of the theorem for constant functions $k$ with the classification $f$ according to the provability of $\varphi_{f}$. Furthermore, this proof method does not depend on whether the original nonparameterized version of the theorem was shown using proof/recursion theory or model-theoretic constructions.

Received March 20, 2013; accepted October 29, 2013

First published online January 6, 2016

2010 Mathematics Subject Classification: Primary 03F30; Secondary 03D20, 03H15

Keywords: finite adjacent Ramsey, Paris-Harrington, Kanamori-McAloon, Ramsey theory, unprovability, independence, phase transitions, Peano arithmetic

(C) 2016 by University of Notre Dame 10.1215/00294527-3452807 


\title{
Phase Transition Results for Three Ramsey-Like Theorems
}

\author{
Florian Pelupessy
}

\begin{abstract}
We classify a sharp phase transition threshold for Friedman's finite adjacent Ramsey theorem. We extend the method for showing this result to two previous classifications involving Ramsey theorem variants: the ParisHarrington theorem and the Kanamori-McAloon theorem. We also provide tools to remove ad hoc arguments from the proofs of phase transition results as much as currently possible.
\end{abstract}

Phase transitions in logic are a recent development in unprovability. The general program, started by Andreas Weiermann, is to classify parameter functions $f: \mathbb{N} \rightarrow \mathbb{N}$ according to the provability of a parameterized theorem $\varphi_{f}$ in a theory $T$. We study these transitions with the goal of gaining a better understanding of unprovability. More details on this program, with an overview of related publications, can be found at Weiermann [16].

In this paper we examine the transition results for three Ramsey theorem variants: Friedman's finite adjacent Ramsey theorem, the Paris-Harrington theorem, and the Kanamori-McAloon theorem. The latter two of these have been studied previously in Weiermann [15] and Carlucci, Lee, and Weiermann [4], but the methods used in the present paper are a natural continuation of the method for the adjacent Ramsey theorem. The emphasis of this method is on connecting the variants $\varphi_{k}$ of the theorem for constant functions $k$ with the classification $f$ according to the provability of $\varphi_{f}$. Furthermore, this proof method does not depend on whether the original nonparameterized version of the theorem was shown using proof/recursion theory or model-theoretic constructions.

Received March 20, 2013; accepted October 29, 2013

First published online January 6, 2016

2010 Mathematics Subject Classification: Primary 03F30; Secondary 03D20, 03H15

Keywords: finite adjacent Ramsey, Paris-Harrington, Kanamori-McAloon, Ramsey theory, unprovability, independence, phase transitions, Peano arithmetic

(C) 2016 by University of Notre Dame 10.1215/00294527-3452807 